\title{
Neural Networks Modeling of Temperature Field Distribution in Hyperthermia
}

\author{
Yung-Yaw Chen, Chi-Hung Chen and Win-Li Lin" \\ Department of Electrical Engineering \& Center of Biomedical Engineering* \\ National Taiwan University, Taipei. Taiwan
}

\begin{abstract}
Hyperthermia is known to be a method of killing tumor cells by heating. Ultrasound transducer is often used to be the heating device. In order to kill the tumor cells and not to injure the normal tissue, the temperature distribution generated by ultrasound nust be predetermined. For a multi-element ultrasound transducer, the phase and the amplitude of the input signal for each elenent can be tuned to generate a suitable temperature distribution to meet the needs of individual treatment. However, direct computation is often time-consuming. While there are also difficulties in solving the ultrasound transducer parameters with a given temperature distribution. In this paper, artificial neural networks are used to learn the relationship between the ultrasound transducer parameters and the temperature distribution, both in the forward and the inverse directions.
\end{abstract}

\section{Introduction}

The effect of hyperthermia is based on the observation that if the tissue temperature is heated to $43^{\circ} \mathrm{C}$ for $30-60$ minutes, the surviving tissue cells will be less than one thousands[1]. Thus, a properly designed temperature distribution could achieve the objective of killing the tumor cells and not injuring normal tissue. Multi-element ultrasound transducer is often used to be the source of heating. The amplitude and phase of the input signal for each element can be tuned to derive a suitable power intensity distribution[2][3]. Then a temperature field can be set up within the tissue 
to perform hyperthermia treatment. The calculation of the temperature filed is very time-consuming and is unrealistic if real-time adjustment of treatment planning is desired. As a result, the neural networks modeling of the ultrasound temperature filed seems to be a solution for determining the relationship between the inputs of the ultrasound transducer and the temperature filed created.

\section{Ultrasound Transducer}

The ultrasound transducer used in this paper has two elements, the inner disk (with diameter $0.75 \mathrm{~cm}$ ) and the outer ring (with diameter $1.5 \mathrm{~cm}$ ). The amplitudes of the input power and the phase between the two elements can be tuned. According to the assumption of uniform tissue characteristics and the circular transducer, temperature distribution is symmetric to the axis that is perpendicular to the transducer surface and is through the transducer center. A typical temperature distribution by direct calculation with the related equations is shown in figure 1 . The sampling distance is $0.075 \mathrm{~cm}$, i.e. with totally $21 \times 200$ points.

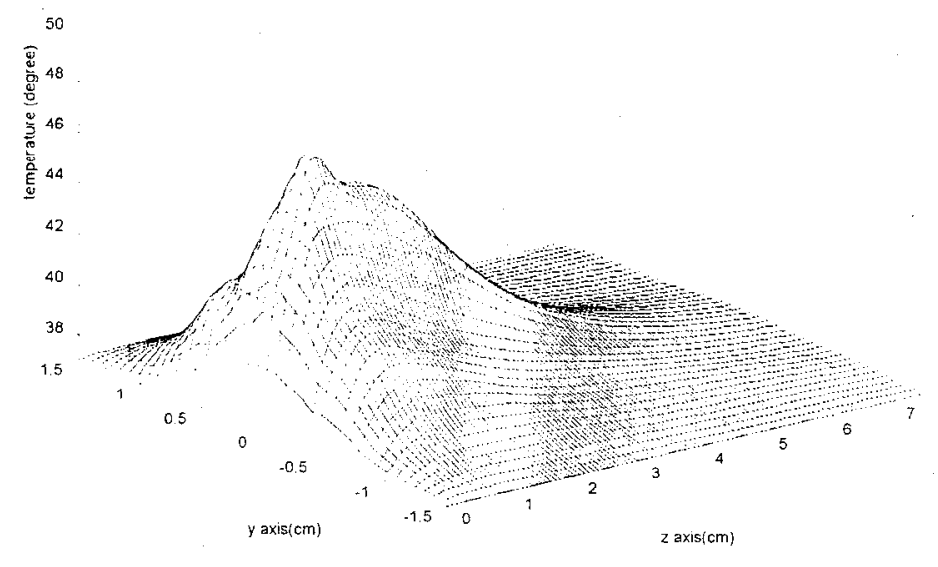

Figure 1 The Typical Temperature Field 


\section{Temperature Field Modeling}

The well-known perceptron-like multi-layer neural networks are used in the modeling of the temperature field in this paper. There is only one hidden layer, other than the input and the output layers. The learning algorithm is based on the backpropagation algorithm[4]. Its learning rate is often determined by trial and error.

\section{Forward Model}

The forward model is designed to emulate the relationship between the transducer inputs and the temperature field. The input parameters of the model are the signal amplitudes for the inner disk and the outer ring, and the their phase difference. The output variable is the temperature distribution (2100 points). Owing to the large number of output variables, 21 neural networks are used to reduced the size of the individual net. 80 data set were calculated to be used as the training data. After training with the training data set, 10 randomly chosen data were used as the testing data sets. The result of the testing data is shown in table 2. The Root-MeanSquare(RMS) errors of the 10 cases demonstrated that the errors are within very acceptable ranges. Actually, the RMS error is less than 0.14 " $\mathrm{C}$ even in the worst case (case 6). Note that the average temperature elevation in percentage is rather high for case 9 , even though its RMS error is only $0.06984^{\circ} \mathrm{C}$. This data may be caused by the fact that its maximum temperature elevation is only $0.94^{\circ} \mathrm{C}$. (The base temperature is $37^{\circ} \mathrm{C}$ in our calculations.)

The above result shows that the idea of using neural networks for temperature field modeling in hyperthermia is feasible. The most important advantage of using the neural networks modeling is really not on its accuracy, but on the its computation speed. The training time for a neural network is usually long. However, its computation of the temperature fields only takes $0.06 \mathrm{sec}$ with a Pentium $90 \mathrm{CPU}$ after training. Such a speed will enable us to consider real-time adjustments of treatment planning, or we can call it "dynamic treatment planning". 


\begin{tabular}{|c|c|c|c|c|c|}
\hline & RMS Error & $\begin{array}{c}\text { Average Temperature } \\
\text { Elevation (\%) }\end{array}$ & $\begin{array}{c}\text { Upper Bound } \\
\text { of Error }\end{array}$ & $\begin{array}{c}\text { Lower Bound } \\
\text { of Error }\end{array}$ & Max. Temp \\
\hline Case 1 & 0.07572 & $1.947 \%(3.222)$ & 0.0915 & -0.3071 & 44.01 \\
\hline Case 2 & 0.06989 & $7.816 \%(0.9075)$ & 0.1739 & -0.1049 & 39.88 \\
\hline Case 3 & 0.11490 & $12.60 \%(0.8341)$ & 0.1984 & -0.4412 & 40.44 \\
\hline Case 4 & 0.07160 & $2.097 \%(3.057)$ & 0.1912 & -0.1636 & 42.38 \\
\hline Case 5 & 0.05257 & $2.211 \%(1.924)$ & 0.06136 & -0.2504 & 41.30 \\
\hline Case 6 & 0.13910 & $4.495 \%(2.495)$ & 0.4486 & -0.2845 & 42.07 \\
\hline Case 7 & 0.05621 & $5.154 \%(0.8556)$ & 0.1900 & -0.05731 & 38.65 \\
\hline Case 8 & 0.04142 & $0.9854 \%(4.303)$ & 0.1414 & -0.07943 & 46.05 \\
\hline Case 9 & 0.06984 & $27.16 \%(0.2741)$ & 0.1735 & -0.004953 & 37.94 \\
\hline Case 10 & 0.03920 & $1.211 \%(3.429)$ & 0.05792 & -0.1276 & 45.04 \\
\hline Average & 0.07896 & & 0.17278 & -0.1821 & \\
\hline
\end{tabular}

Table 1 Testing Result for the Forward Model

\section{Inverse Model}

The inverse model is designed to establish the relationship between the temperature field and the transducer inputs, i.e. to determine the proper transducer inputs which can form a desired temperature filed. The structure of the neural network used is similar to the forward case, except the inputs and the outputs are reversed. For the inverse case, it is observed that the number of data for the temperature field needed for establishing the relationship is not as high as in the forward case. Instead of using 2100 points, only $4 \times 15(60)$ points are used.

The result with the testing data set is shown in table 2. As we can see that the error of the estimated particle velocity seems to be related to the magnitude of the velocity. The higher the particle velocity is, the smaller the error. While in case 4 , when the velocity for the inner disk is much smaller than the velocity of the outer ring, the 
temperature filed is dominated by the outer ring and the phase difference becomes insignificant. As a result, the error for the phase difference is large. However, we will show in below that the phase difference plays very little role in such a case and is literally insignificant. In case 2 and case 9 , it is also observed that the phase differences are out of the range of training set and the error become large.

\begin{tabular}{|l|l:l|l|l|l|l|}
\hline & $\begin{array}{l}\text { Particle } \\
\text { Velocity of } \\
\text { Outer Ring }\end{array}$ & Reference & $\begin{array}{l}\text { Particle } \\
\text { Velocity of } \\
\text { Inner Disk }\end{array}$ & Reference & Difference & Reference \\
\hline Case 1 & 3.79 & 3.84 & 1.55 & 1.76 & $1.20 \pi$ & $1.06 \pi$ \\
\hline Case 2 & 0.37 & 0.80 & 2.80 & 2.64 & $1.43 \pi$ & $0.18 \pi$ \\
\hline Case 3 & 0.08 & 0.92 & 2.80 & 2.88 & $1.49 \pi$ & $1.30 \pi$ \\
\hline Case 4 & 3.78 & 3.76 & 0.09 & 0.08 & $1.44 \pi$ & $0.84 \pi$ \\
\hline Case 5 & 2.87 & 2.92 & 1.96 & 1.96 & $1.42 \pi$ & $1.40 \pi$ \\
\hline Case 6 & 2.82 & 2.72 & 2.84 & 3.00 & $1.86 \pi$ & $1.82 \pi$ \\
\hline Case 7 & 1.94 & 1.96 & 0.32 & 1.08 & $1.65 \pi$ & $1.52 \pi$ \\
\hline Case 8 & 3.99 & 4.00 & 2.32 & 2.24 & $0.48 \pi$ & $0.52 \pi$ \\
\hline Case 9 & 0.05 & 0.32 & 0.97 & 1.56 & $1.53 \pi$ & $0.10 \pi$ \\
\hline Case 10 & 3.69 & 3.72 & 3.18 & 3.16 & $1.49 \pi$ & $1.48 \pi$ \\
\hline
\end{tabular}

Table 2 Testing Result for the Inverse Model

It is pretty obvious that different transducer inputs may construct the same temperature field within our error tolerance. Therefore, the inverse model may be usable even if its outputs deviate from the reference values. As a matter of fact. the inverse model is "correct" if the transducer inputs calculated by the neural networks can form the desired temperature field. Two different verification approaches are used to verify the data in table 2. The ID method is to compare the reference temperature field with the filed derived through inverse model plus direct 
computations. The IF method is to compare the reference temperature field with the filed derived through inverse model plus the forward model. The average RMS error for the ID method is 0.14899 and is 0.16455 for the IF method. To be noticed that the IF method has an error only slightly higher than the ID method and is still in the acceptable range.

\section{Conclusion}

The forward model and the inverse model of the temperature field in hyperthermia are shown in this paper. Multi-layer neural networks are adopted for modeling and have the advantage of real-time computation after proper training. Both models achieve excellent performance with very acceptable accuracy. Although the inverse model may not derive the exact reference values, its corresponding temperature fields are still very similar to the reference ones. The fast computation of the temperature field in hyperthermia creates the possibility of the real-time adjustment of the treatment planning, i.e. as we call it, the dynamic treatment planning.

\section{Reference}

[1] N. Auersperg, "Differential Heat Sensitivity of Cells in Tissue Culture", Nature, vol. 209, pp. 415-416, 1966.

[2] Chia-Jong Chang,"Ultrasound Power Intensity Distributions of Annular Array Transducer Used in Hyperthermia Cancer Therapy", MS Thesis, NTU ME Dept. 1995.-

[3] Yung-Yaw Chen, Da-Ching Lu, Win-Li Lin and Chi-Hung Chen, "Estimation of Power Intensity Distribution with Artificial Neural Network for Ultrasound Hyperthermia", Biomed Eng Appl Basis Comm, pp. 43-48, Feb. 1996.

[4] Simon Haykin, Neural Networks: A Comprehensive Foundation, New York: Macmillan, 1994. 